\title{
BMJ Open Acupuncture for treating sciatica: a systematic review protocol
}

\author{
Zongshi Qin, ${ }^{1,2}$ Xiaoxu Liu, ${ }^{1,2}$ Qin Yao, ${ }^{1,2}$ Yanbing Zhai, ${ }^{1,2}$ Zhishun Liu ${ }^{1}$
}

To cite: Qin Z, Liu X, Yao Q, et al. Acupuncture for treating sciatica: a systematic review protocol. BMJ Open 2015;5: e007498. doi:10.1136/ bmjopen-2014-007498

- Prepublication history for this paper is available online. To view these files please visit the journal online (http://dx.doi.org/10.1136/ bmjopen-2014-007498).

Received 19 December 2014 Revised 5 March 2015 Accepted 20 March 2015
CrossMark

\footnotetext{
${ }^{1}$ Department of Acupuncture, Guang'anmen Hospital, China Academy of Chinese Medical Sciences, Beijing, China

${ }^{2}$ Beijing University of Chinese Medicine, Beijing, China
}

Correspondence to Professor Zhishun Liu; liuzhishun@aliyun.com

\section{ABSTRACT}

Introduction: This systematic review aims to assess the effectiveness and safety of acupuncture for treating sciatica.

Methods: The following nine databases will be searched from their inception to 30 October 2014: MEDLINE, EMBASE, the Cochrane Central Register of Controlled Trials (CENTRAL), the Chinese Biomedical Literature Database (CBM), the Chinese Medical Current Content (CMCC), the Chinese Scientific Journal Database (VIP database), the Wan-Fang Database, the China National Knowledge Infrastructure (CNKI) and Citation Information by National Institute of Informatics (CiNii). Randomised controlled trials (RCTs) of acupuncture for sciatica in English, Chinese or Japanese without restriction of publication status will be included. Two researchers will independently undertake study selection, extraction of data and assessment of study quality. Meta-analysis will be conducted after screening of studies. Data will be analysed using risk ratio for dichotomous data, and standardised mean difference or weighted mean difference for continuous data.

Dissemination: This systematic review will be disseminated electronically through a peer-reviewed publication or conference presentations.

Trial registration number: PROSPERO CRD42014015001.

\section{INTRODUCTION}

Sciatica is a common neuralgia characterised by pain radiating into the leg; it is usually caused by nerve root compression and irritation or inflammation of the sciatic nerve, and is often accompanied by lower back pain and neurological deficits in the lower limb. ${ }^{1}$ The pain is often associated with tingling, numbness and weakness of the leg; it may be sudden in onset and then persist for days or weeks. $^{2}$ Frymoyer $^{3}$ reported that sciatica is very common, with a lifetime incidence varying from $13 \%$ to $40 \%$ and a corresponding annual incidence of sciatica episodes of $1-5 \%{ }^{4}$

Sciatica commonly affects people between the ages of 30 and 50 years, with the pain of sciatica significantly damaging health. ${ }^{5}$ Thus,

\section{Strengths and limitations of this study}

The effectiveness of conservative therapy for sciatica is uncertain, and acupuncture may provide an effective alternative treatment method. To the best of the authors' knowledge, there is currently no systematic review published in English related to acupuncture for sciatica. The results of this systematic review will help clinicians make decisions on treating sciatica, and also help patients seeking further treatment options.

- One limitation of this systematic review is that, owing to the language barrier, trials in only three languages can be included. Hence, relevant studies published in other languages might be missed.

- Another limitation is that the different forms of acupuncture therapy and quality of methodologies in the included studies may cause significant heterogeneity.

according to a report from Younes, ${ }^{6}$ sciatica in Tunisia has become a major cause of work absenteeism and a financial burden to society. Previous research has indicated that $60 \%$ of patients with sciatica suffer from a mild disability. On the basis of questionnaires (given at the 3rd through 12th months of symptom exhibition), it is believed that $30 \%$ of patients live with sciatica for more than 1 year, which results in an obvious decrease in the quality of life. ${ }^{7}$ The current management of sciatica can be classified into pharmacological and non-pharmacological treatment. One article supports the effectiveness of non-opioid medication, epidural injections and disc surgery. It also suggests that spinal manipulation, acupuncture and experimental treatments, such as antiinflammatory biological agents, may be considered. ${ }^{8}$ The use of pharmacological products such as anaesthetics or corticosteroids has associated adverse effects including sedation, dizziness, ataxia and nausea, and their effectiveness decreases with long-term use. ${ }^{9}$ Although according to the prior systematic review epidural injections are more effective for pain reduction than non-opioids, ${ }^{8}$ related 
adverse effects to epidural injections have been reported. ${ }^{10-12}$ To date, there is no strong evidence-based medicine proving that non-pharmacological conservative treatment of Western medicine is effective ${ }^{13}{ }^{14}$; also, surgical procedures are invasive, expensive and may cause neurological complications. ${ }^{15}$

In China, sciatica is a primary cause for hospitalisation and it is commonly used for managing neuralgia pain. ${ }^{16}$ Acupuncture is reported to be effective in treating many types of musculoskeletal pain including lower back pain, ${ }^{17}$ fibromyalgia, ${ }^{18}$ osteoarthritis ${ }^{19}$ and sciatica. ${ }^{20-24}$ However, the ability of acupuncture to successfully manage sciatica, either as a monotherapy or as an adjunct to Western medical care, remains unclear.

This systematic review aims to assess the effectiveness and safety of acupuncture for treating sciatica. To this end, we will pose the following question: What is the comparative effectiveness and safety of acupuncture compared with sham acupuncture, usual care or no treatment to reduce pain intensity in patients diagnosed with sciatica? Is there a definitive advantage of acupuncture compared with Western medication? With the resulting evaluation aiming to help clinicians make decisions on treating sciatica, and to help patients seeking further treatment options.

\section{METHODS AND ANALYSIS}

Criteria for inclusion of studies in this review:

\section{Types of studies}

Only RCTs will be included; quasi-RCTs and randomised cross-over studies will be excluded. Blinding will not be considered because of the characteristics of acupuncture treatment.

\section{Types of participants}

Patients with sciatica will be included, including those diagnosed with sciatica synonyms such as radiculopathy, nerve root compromise, nerve root compression, nerve root pain and pain radiating below the knee. There will be no restriction on sex, age or the intensity or duration of symptoms.

Patients with acute infection, caudal equina syndrome, primary spinal stenosis and lower back pain without sciatica will be excluded.

\section{Types of interventions}

- Any type of invasive acupuncture will be included, such as acupuncture, electro-acupuncture, elongated needle acupuncture, three-edged needle acupuncture, fire needling, auricular acupuncture, abdominal acupuncture, warm acupuncture and pyonex. Control interventions may include general care, sham acupuncture/placebo and waiting list care.

- Acupuncture versus other Western medicine treatment will be included.
- Acupuncture plus another Western medicine treatment versus the same Western medicine treatment alone will be included.

- RCTs comparing two different types of acupuncture will be excluded.

- Acupuncture treatment without needle insertion (eg, acupressure, laser acupuncture and electrical stimulation) will be excluded.

- Acupuncture combined with Chinese medicine, acupoint injection and/or needle knife will be excluded.

\section{Types of outcome assessments}

Primary outcomes

- Pain intensity. Any validated measurement scales will be included (eg, visual analogue scale, numeric rating scale, short-form McGill Pain Questionnaire (SF-MPQ)).

- Global assessment (the proportion of patients improved or cured).

Secondary outcomes

- Quality of life, for example, as assessed using the Medical Outcomes Study 36-item Short Form health survey (SF-36).

- Physical examinations.

- Patient satisfaction.

- Adverse effects.

\section{Search methods for identification of studies}

A search strategy will be used and conducted according to the Cochrane handbook guidelines. ${ }^{25}$ The following nine databases will be searched from their inception to 30 October 2014: MEDLINE, EMBASE, the Cochrane Central Register of Controlled Trials (CENTRAL), the Chinese Biomedical Literature Database (CBM), the Chinese Medical Current Content (CMCC), the Chinese Scientific Journal Database (VIP database), the Wan-Fang Database, the China National Knowledge Infrastructure (CNKI) and Citation Information by National Institute of Informatics (CiNii). The search strategy is based on the guidance of the Cochrane handbook. The key words include 'sciatica', 'sciatic neuralgia', 'discogenic sciatica', 'disc herniation-induced sciatica', 'bilateral sciatica', 'acupuncture', 'electro-acupuncture', 'elongated needle', 'three-edged needle', 'fire needling', 'auricular acupuncture', 'abdominal acupuncture' and 'pyonex'.

The strategy for searching the PUBMED database is shown in table 1 . This search strategy will also be applied to the other electronic databases.

\section{Data collection and analysis}

\section{Selection of studies}

Two authors (XL and YZ) will screen the title and abstracts of all the articles to confirm that they contain eligible trials, with the full text to be reviewed if necessary. Any disagreement during the selection of studies will be discussed and decided by a third author (ZL). Details of the selection process are shown in the PRISMA flow chart (figure 1). 
Table 1 Search strategy used in the PubMed database

\begin{tabular}{ll} 
Number & Search items \\
\hline 1 & randomized controlled trial.pt \\
2 & controlled clinical trial.pt \\
3 & randomized.ti,ab \\
4 & randomly.ti,ab \\
5 & groups.ti,ab \\
6 & trial.ti,ab \\
7 & or 1-6 \\
8 & acupuncture.ti,ab \\
9 & electro-acupuncture.ti,ab \\
10 & elongated needle.ti,ab \\
11 & three edged needle.ti,ab \\
12 & (fire needle or warming needle).ti,ab \\
13 & auricular acupuncture.ti,ab \\
14 & abdominal acupuncture.ti,ab \\
15 & warm acupuncture.ti,ab \\
16 & pyonex.ti,ab \\
17 & or 8-16 \\
18 & sciatica.ti,ab \\
19 & sciatic neuralgia.ti,ab \\
20 & discogenic sciatica.ti,ab \\
21 & bilateral sciatica.ti,ab \\
22 & disc herniation-induced sciatica.ti,ab \\
23 & or 18-22 \\
24 & 7 and 17 and 23 \\
\hline & be modfied as required for other
\end{tabular}

This search strategy will be modified as required for other electronic databases.

\section{Data extraction and management}

A data extraction form will be used to collect data. A small scope trial will be done before the systematic review is conducted to confirm that there is no obvious divergence between those collecting data. Two authors (XL and $\mathrm{YZ}$ ) will independently extract the data and take the following aspects into consideration: general information (name and year of publication, date of extraction, title of study and author's publication details), study characteristics, eligibility criteria, interventions, outcome measurements, duration, adverse events, results and the type of needle used. All searched studies will be inputted to EndNote software that can assist reviews to manage data and pick up duplicate publications; when two or more publications described a single trial, we included only one report. These data will then be entered into RevMan V.5.3.3 software for analysis. Any disagreement will be discussed and finally decided on by a third author (ZL).

\section{Data items}

We will extract the information of each study, including the type of control used, frequency and duration of treatment, patient characteristics (age, gender, duration of symptoms, type of sciatica), trial design, trial size, duration of follow-up, type and source of financial support, if appropriate.

\section{Assessment of risk of bias in included studies}

The risk of bias assessment will be based on the Cochrane Collaboration Risk of Bias Tool. ${ }^{26}$ Two authors
(QY and YZ) will independently evaluate methodological quality using the following seven domains: random sequence generation, allocation concealment, blinding of participants and personnel, blinding of outcome assessment, incomplete outcome data, selective reporting and other bias. Other sources of bias may be caused by the different types of needles used, the duration of sciatica, the length of therapy and the age of patients. Taking these domains into account, each trial will be categorised into low risk, high risk and unclear risk. Any disagreements will be discussed and resolved by a third author (ZL).

\section{Confidence in cumulative estimate}

Details of acupuncture and control interventions were extracted on the basis of the revised Standard for Reporting Interventions in Clinical Trials of Acupuncture (STRICTA), ${ }^{27}$ a checklist that was intended for use in conjunction with CONSORT and can estimate RCTs of acupuncture, include acupuncture rationale, needling details, treatment regimen, co-intervention, control interventions and treating background.

\section{Measurement of treatment effect}

Analysis will be based on available data of included studies. For dichotomous data, the risk ratio (RR) will be calculated with 95\% CIs. For continuous variables, means and SDs will be used to calculate a mean difference with a $95 \%$ CI.

\section{Dealing with missing data}

The listed corresponding author will be contacted to try and obtain any missing information from their trial. If it is impossible to obtain the data, the study will be excluded from the data synthesis.

\section{Assessment of heterogeneity}

Before combining the statistics, tests for heterogeneity will be used to judge the homogeneity of the studies. If the resulting $\mathrm{p}$ value exceeds 0.1 , indicating significant heterogeneity among trials, the reasons leading to heterogeneity will be analysed and subgroup analysis will be conducted.

\section{Assessment of reporting biases}

A funnel plot will be used to assess the reporting biases if 10 or more trials are included in a meta-analysis.

\section{Data synthesis}

If meta-analysis can be conducted, RevMan V.5.3.3 software will be used to combine the RR with $95 \%$ CIs for dichotomous outcomes and the weighted mean difference or standardised mean difference with $95 \%$ CIs for continuous data. If the result of the test for heterogeneity results in $\mathrm{p}>0.1$, the fixed-effect model will be used to combine the data; if $\mathrm{p}<0.1$, the random-effect model will be used. If the data will not be suitable for combining quantitatively, in the condition, a systematic narrative 


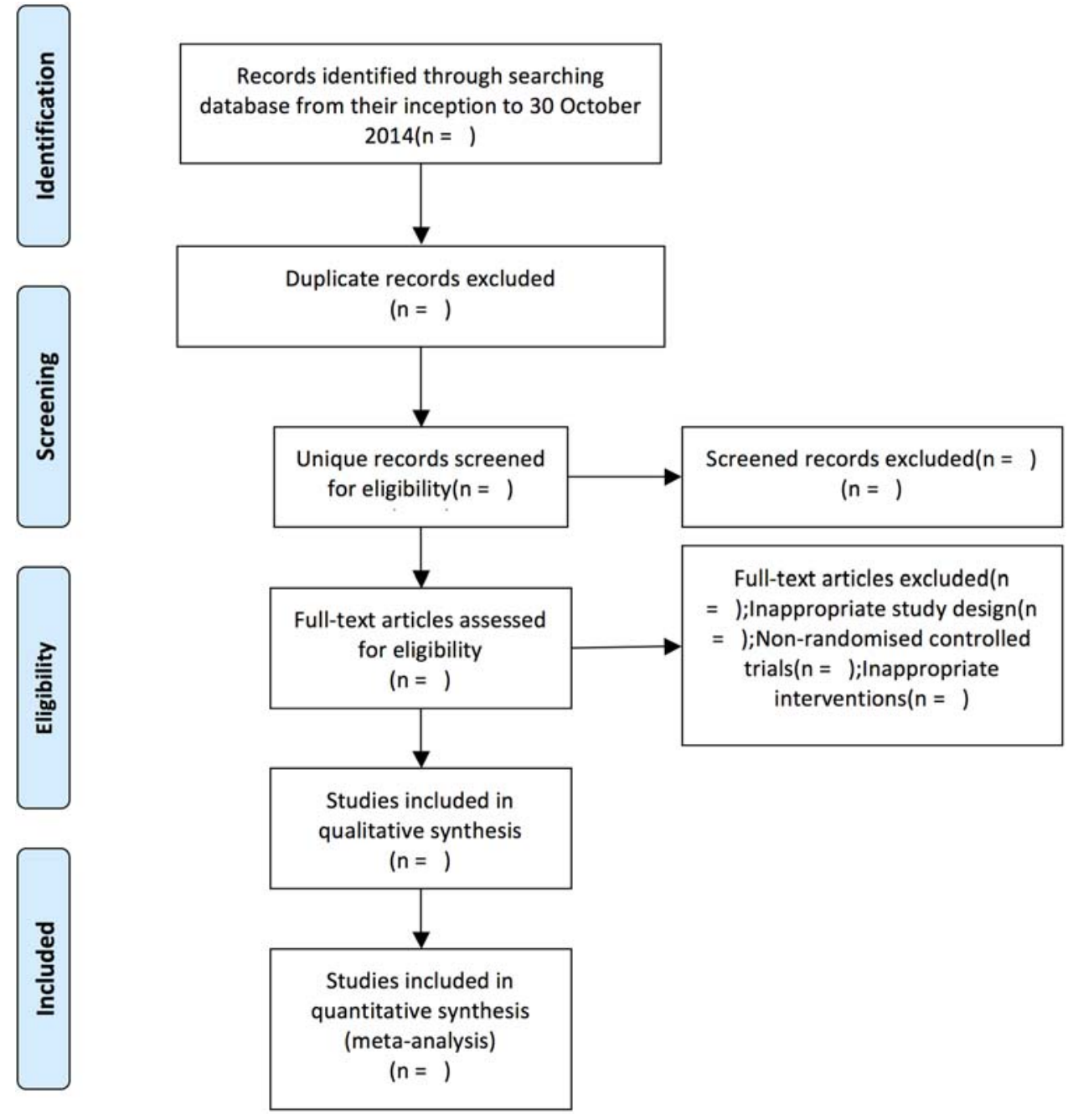

Figure 1 Study flow diagram.

synthesis will be provided with the information that presented in the text to summarise and explain the characteristics and findings of the included studies.

\section{Subgroup analysis}

The following subgroup analyses will be conducted to assess the heterogeneity of the studies:

Clinical considerations

- Acupuncture versus sham acupuncture,

- Types of sciatica (non-discogenic sciatica vs discogenic sciatica).

Methodological considerations

- Trials with unclear or high risk of bias.

\section{Sensitivity analysis}

If the test for heterogeneity $p$ value is less than 0.1 after the data extraction has been checked and subgroup analyses conducted, the low-quality studies will be excluded and the meta-analysis will be conducted again.

\section{Ethics and dissemination}

This systematic review will not use data from individual patients to protect privacy, and the results of this systematic review will be disseminated only in a peerreviewed publication.

\section{DISCUSSION}

Sciatica causes significant suffering for the individual, yet most of the currently available treatment options are not adequate to control pain. Pharmacological methods have associated adverse effects, while surgery is expensive and is not appropriate for every patient. Acupuncture has been used for 3000 years in China and is generally regarded as a safe and effective measure to alleviate pain. However, when the effectiveness of acupuncture for a condition remains unclear, it is difficult for clinicians to make appropriate recommendations. The mechanism of acupuncture analgesia is gradually becoming known. $\mathrm{Han}^{28}$ found that acupuncture can promote release of neurotransmitter such as 5hydroxytryptamine and in addition it generates neuropeptide through electrical stimulation of different frequencies that has significantly effect to pain reduction.

This is a protocol for a systematic review that aims to assess the safety and effectiveness of acupuncture for 
sciatica. As there has been no prior systematic review related to acupuncture for sciatica published in English, we hope this systematic review will help clinicians make decisions in practice and promote the progress of acupuncture research.

This review has some potential limitations. Different forms of acupuncture therapies and the quality of methodology in included trials may cause significant heterogeneity. There also may be some relevant studies missed, as only studies published in English, Chinese and Japanese will be included.

Acknowledgements At the point of finishing this paper, the authors would like to express their sincere thanks to all those who have lent them a helping hand over the course of the writing of this paper. They also take this opportunity to show their sincere gratitude to Mr Brendan Melchiorri, who has given them so much useful advice on writing and tried his best to improve their paper.

Contributors ZL and ZQ contributed to the conception of the study. The manuscript protocol was drafted by $Z Q$ and revised by $Q Y$. The search strategy was developed by all the authors and will be performed by $Y Z$ and $\mathrm{XL}$, who will also independently screen the potential studies, extract data from the included studies, assess the risk of bias and complete the data synthesis. ZL will arbitrate in cases of disagreement and ensure the absence of errors. All authors approved the publication of the protocol.

Competing interests None declared.

Provenance and peer review Not commissioned; externally peer reviewed.

Open Access This is an Open Access article distributed in accordance with the Creative Commons Attribution Non Commercial (CC BY-NC 4.0) license, which permits others to distribute, remix, adapt, build upon this work noncommercially, and license their derivative works on different terms, provided the original work is properly cited and the use is non-commercial. See: http:// creativecommons.org/licenses/by-nc/4.0/

\section{REFERENCES}

1. Stafford MA, Peng P, Hill DA, et al. Sciatica: a review of history, epidemiology, pathogenesis, and the role of epidural steroid injection in management. Br J Anaesth 2007;99:461-73.

2. Mathieson S, Maher CG, McLachlan AJ, et al. PRECISEpregabalin in addition to usual care for sciatica: study protocol for a randomised controlled trial. Trials 2013;14:213.

3. Frymoyer JW. Lumbar disk disease: epidemiology. Instr Course Lect 1992;41:217-23.

4. Frymoyer JW. Back pain and sciatica. $N$ Engl $J$ Med 1988;318:291-300.

5. Konstantinou K, Dunn KM. Sciatica: review of epidemiological studies and prevalence estimates. Spine 2008;33:2464-72.

6. Younes M, Bejia I, Aguir Z, et al. Prevalence and risk factors of disk-related sciatica in an urban population in Tunisia. Joint Bone Spine 2006;73:538-42.

7. Weber H, Holme I, Amlie E. The natural course of acute sciatica with nerve root symptoms in a double-blind placebo-controlled trial evaluating the effect of piroxicam. Spine 1993;18:1433-8.
8. Lewis RA, Willams NH, Sutton AJ, et al. Comparative clinical effectiveness of management strategies for sciatica: systematic review and network meta-analyses. Spine J 2013. [Epub ahead of print 4 Oct 2013].

9. Valat JP, Giraudeau B, Rozenberg S, et al. Epidural corticosteroid injections for sciatica: a randomised, double blind, controlled clinical trial. Ann Rheum Dis 2003;62:639-43.

10. Carette S, Leclaire R, Marcoux S, et al. Epidural corticosteroid injections for sciatica due to herniated nucleus pulposus. $N$ Engl J Med 1997;336:1634-40.

11. Finckh A, Zufferey $P$, Schurch MA, et al. Short-term efficacy of intravenous pulse glucocorticoids in acute discogenic sciatica. A randomized controlled trial. Spine 2006;31:377-81.

12. Korhonen T, Karppinen J, Paimela L, et al. The treatment of disc herniation-induced sciatica with infliximab: results of a randomized, controlled, 3-month follow-up study. Spine 2005;30:2724-8.

13. Atlas SJ, Keller RB, WU YA, et al. Long-term outcomes of surgical and nonsurgical management of lumbar spinal stenosis: 8 to 10 year results from the maine lumbar spine study. Spine 2005;30:936-43.

14. Legrand $E$, Bouvard $B$, Audran $M$, et al. Sciatica from disk herniation: medical treatment or surgery? Joint Bone Spine 2007;74:530-5.

15. Van Tulder MW, Koes B, Seitsalo S, et al. Outcome of invasive treatment modalities on back pain and sciatica: an evidence-based review. Eur Spine J 2006;15:S82-92.

16. Wang SZ, Wu GY, Huang Y, et al. Integrated traditional Chinese and western medicine treatment overview for sciatica. HuNan J Tradit Chin Med 2014;30:133-4.

17. Lee JH, Choi TY, Lee MS, et al. Acupuncture for acute low back pain: a systematic review. Clin J Pain 2013;29:172-85.

18. Iannuccelli C, Mannocci F, Guzzo MP, et al. Complementary treatment in fibromyalgia: combination of somatic and abdominal acupuncture. Clin Exp Rheumatol 2012;30:112-16.

19. Witt C, Brinkhaus B, Jena $S$, et al. Acupuncture in patients with osteoarthritis of the knee: a randomised trial. Lancet 2005;366:136-43.

20. Duplan B, Cabanel G, Piton JL, et al. Acupuncture and sciatica in the acute phase. Double-blind study of 30 cases. Sem Hop 1983;59:3109-14.

21. Zhang P. Essentials for the acupuncture treatment of radicular sciatica. J Tradit Chin Med 2003;23:237.

22. Li J, Dong JC, Yue JJ. Effects of acupuncture on default mode network images of chronic sciatica patients in the resting network state. Zhongguo Zhong Xi Yi Jie He Za Zhi 2012;32:1624-7.

23. Chen MR, Wang $P$, Cheng $G$, et al. The warming acupuncture for treatment of sciatica in 30 cases. J Tradit Chin Med 2009;29:50-3.

24. Wang BX, La JL. Therapeutic effects of electro-acupuncture and diclofenic on herniation of lumbar intervertebral disc. Zhongguo Linchuang Kangfu 2004;8:3413-15.

25. Higgins JP, Green S. Cochrane handbook for systematic reviews of interventions, version 5.1.0 [updated March 2011]. The Cochrane Collaboration, 2011.

26. Higgins JP, Altman DG, Gotzsche PC, et al. Cochrane Bias Methods Group; Cochrane Statistical Methods Group: the Cochrane Collaboration's tool for assessing risk of bias in randomised trials. BMJ 2011;343:d5928.

27. MacPherson H, Altman DG. Hammerschlag, R, et al., Revised STandards for Reporting Interventions in Clinical Trials of Acupuncture (STRICTA): extending the CONSORT statement. PLOS Med 2010;7:e1000261.

28. Han JS. Acupuncture: neuropeptide release produced by electrical stimulation of different frequencies. Trends Neurosci 2003;26:17-22. 